\title{
A Histone Acetylation Switch Regulates H2A.Z Deposition by the SWR-C Remodeling Enzyme
}

\author{
Shinya Watanabe ${ }^{1}$, Marta Radman-Livaja ${ }^{2}$, Oliver J. Rando ${ }^{2}$, and Craig L. Peterson ${ }^{1, *}$ \\ ${ }^{1}$ Program in Molecular Medicine, 373 Plantation Street, University of Massachusetts Medical \\ School, Worcester, MA 01605, USA \\ ${ }^{2}$ Department of Biochemistry and Molecular Pharmacology, 364 Plantation Street, University of \\ Massachusetts Medical School, Worcester, MA 01605, USA
}

\begin{abstract}
The histone variant H2A.Z plays key roles in gene expression, DNA repair, and centromere function. H2A.Z deposition is controlled by SWR-C chromatin remodeling enzymes that catalyze the nucleosomal exchange of canonical H2A with H2A.Z. Here we report that acetylation of histone $\mathrm{H} 3$ on lysine $56(\mathrm{H} 3-\mathrm{K} 56 \mathrm{Ac})$ alters the substrate specificity of SWR-C, leading to promiscuous dimer exchange in which either $\mathrm{H} 2 \mathrm{~A} . \mathrm{Z}$ or $\mathrm{H} 2 \mathrm{~A}$ can be exchanged from nucleosomes. This result was confirmed in vivo, where genome-wide analysis demonstrated widespread decreases in H2A.Z levels in yeast mutants with hyperacetylated H3K56. Our work also suggests that a conserved SWR-C subunit may function as a "lock" that prevents removal of H2A.Z from nucleosomes. Our study identifies a histone modification that regulates a chromatin remodeling reaction and provides insights into how histone variants and nucleosome turnover can be controlled by chromatin regulators.
\end{abstract}

The H2A.Z histone variant is typically found within nucleosomes that flank promoters of genes transcribed by RNA polymerase II, as well as nucleosomes that flank chromatin boundary elements, centromeres, and replication origins (1-3). These nucleosomes also exhibit rapid, replication-independent turnover, which is thought to function in erasing histone marks, preventing the spread of chromatin states, and ensuring general plasticity of the epigenome $(4,5)$. H2A.Z appears to enhance rapid turnover of promoter proximal nucleosomes in yeast (4), and nucleosomes subject to rapid turnover kinetics are also enriched for histone $\mathrm{H} 3$ acetylated at lysine $56(\mathrm{H} 3-\mathrm{K} 56 \mathrm{Ac})(6)$. H3-K56Ac is also required for enhanced turnover of promoter nucleosomes $(6,7)$. Recent work indicates that vertebrate gene promoters are also enriched in nucleosomes harboring both H2A.Z and H3-K56Ac, suggesting a conserved regulatory relationship $(2,8,9)$. How they cooperate in this process, though, is not clear.

To test whether nucleosomes that harbor both $\mathrm{H} 2 \mathrm{~A} . \mathrm{Z}$ and H3-K56Ac are inherently unstable, recombinant yeast mononucleosomes were immobilized on streptavidin beads, and nucleosome stability was monitored after exposure to increasing salt concentration (fig. S1). Nucleosomes were reconstituted with either $\mathrm{H} 2 \mathrm{~A} / \mathrm{H} 2 \mathrm{~B}$ or $\mathrm{H} 2 \mathrm{~A} . \mathrm{Z} / \mathrm{H} 2 \mathrm{~B}$ dimers, and with histone $\mathrm{H} 3$ that contained either a lysine at position 56 or a glutamine residue to mimic acetylation (H3-K56Q). H3-K56Q had no detectable effect on the stability of the H2A/H2B

Copyright 2013 by the American Association for the Advancement of Science; all rights reserved.

*Corresponding author. craig.peterson@umassmed.edu.

Supplementary Materials

www.sciencemag.org/cgi/content/full/340/6129/195/DC1 
dimer-H3/H4 tetramer interaction (fig. S1, top panels) $(10,11)$. By contrast, incorporation of H2A.Z led to a decreased salt stability of both H3 and H3-K56Q mono-nucleosomes (fig. S1, bottom left panel) (12). However, the combination of H2A.Z and H3-K56Q did not further decrease stability (fig. S1, bottom right panel), indicating that this $\mathrm{H} 3$ modification does not itself contribute to marked instability of nucleosomes.

The conserved SWR-C chromatin remodeling enzyme controls H2A.Z deposition in yeast $(13,14)$, and so we next tested whether H3-K56Ac might regulate its histone exchange activity. Recombinant yeast $\mathrm{H} 2 \mathrm{~A}$ mononucleosomes that harbored either $\mathrm{H} 3-\mathrm{K} 56$ or $\mathrm{H} 3-$ K56Q were incubated with purified SWR-C, recombinant H2A.Z/H2B dimers, and adenosine $5^{\prime}$-triphosphate (ATP), and then histone exchange was quantified by a Western blot assay, probing for different epitope-tagged, H2A histones. The integrity of the mononucleosome was analyzed by both Western blotting for $\mathrm{H} 3$ and by visualizing DNA (Fig. 1). SWR-C catalyzed robust deposition of H2A.Z when incubated with the wild-type H2A nucleosomes (14). By contrast, nearly $80 \%$ less H2A.Z was deposited by SWR-C when incubated with the H3-K56Q substrate (Fig. 1A and fig. S2).

SWR-C-catalyzed dimer exchange involves at least two coupled steps-ATP-dependent eviction of the $\mathrm{H} 2 \mathrm{~A} / \mathrm{H} 2 \mathrm{~B}$ dimer from the nucleosome, followed by deposition of $\mathrm{H} 2 \mathrm{~A} . \mathrm{Z} /$ $\mathrm{H} 2 \mathrm{~B}$ (15). We predicted that H3-K56Ac might facilitate both the forward and reverse reactions and might display altered substrate specificity more like that of the related INO80 enzyme (16). We incubated SWR-C with H2A.Z nucleosomes, ATP, and H2A/H2B dimers and found that SWR-C had no effect on the histone composition of the wild-type H2A.Z nucleosome, as expected (Fig. 1B) $(14,16)$. By contrast, SWR-C showed robust eviction of nucleosomal H2A.Z when the nucleosome harbored H3-K56Q (Fig. 1B and fig. S3).

Furthermore, SWR-C catalyzed the ATP-dependent incorporation of H2A when incubated with the H2A.Z/H3-K56Q mononucleosome (Fig. 1B). H2A.Z exchange was efficient, with nearly 30\% H2A replacement (Fig. 1C). H3-K56Q also stimulated a low level of H2A.Z exchange in the absence of SWR-C, indicating that this modification may poise the H2A.Z nucleosome for exchange events (Fig. 1B), perhaps due to enhanced breathing of nucleosomal DNA (11). Robust H2A.Z exchange reaction was dependent on the concentration of SWR-C (fig. S3), ATP, and the time of incubation (fig. S4). H3-K56Q also enhanced the H2A.Z replacement activity of the related INO80 enzyme (Fig. 1B and fig. S5). These effects of H3-K56Q were not due to alterations in the nucleosome binding affinity of the SWR-C or INO80 enzymes (fig. S6). We further examined whether the alterations in SWR-C could also be observed with bona fide H3-K56Ac mononucleosomes (fig. S7) that were incubated with SWR-C in the presence of $\mathrm{H} 2 \mathrm{~A} / \mathrm{H} 2 \mathrm{~B}$ dimers (Fig. $1 \mathrm{D}$ and fig. S8). SWR-C catalyzed the ATP-dependent incorporation of H2A, similar to our results with an H2A.Z nucleosome harboring H3-K56Q.

The substrate specificity of the SWR-C dimer-exchange reaction is reflected by the adenosine triphosphatase (ATPase) properties of SWR-C, as an H2A nucleosome, but not an H2A.Z nucleosome, stimulates the ATPase activity of SWR-C (15). Because ATPdependent remodeling enzymes are DNA-stimulated ATPases, these results suggest that SWR-C productively interacts only with the nucleosomal DNA of an $\mathrm{H} 2 \mathrm{~A}$ nucleosome, consistent with the dimer exchange specificity of SWR-C. To determine if H3-K56Q alters the ATPase properties of SWR-C, ATPase assays were performed with wild-type and H3K56Q nucleosomes. The ATPase activity of SWR-C was stimulated by an H2A nucleosome, but no stimulation was observed with the H2A.Z nucleosome (Fig. 2A). The addition of free H2A.Z/H2B dimers led to a further stimulation (15). By contrast, nucleosomal incorporation of H3-K56Q led to equal ATPase stimulation by both the H2A and H2A.Z nucleosomes, and addition of free dimers had no effect (Fig. 2B). 
The Swc2p subunit of SWR-C binds to H2A.Z, and Swc2p is required for deposition of H2A.Z in vitro (17) and in vivo (13), suggesting that Swc2p functions during the H2A.Z deposition step of the dimer-exchange reaction, presumably by binding and delivering H2A.Z (17). We hypothesized that Swc2p might also function at the end of the reaction cycle, functioning as a molecular "lock" that binds to H2A.Z and prevents SWR-C from removing the newly incorporated H2A.Z.

SWR-C was purified from a $s w c 2 \Delta$ strain, yielding a SWR-C that lacks the Swc2p and Swc3p subunits and was depleted for Arp6 and Swc6 (fig. S9). The Swc2 $\Delta$ subcomplex was unable to deposit H2A.Z into an H2A nucleosome (fig. S10) (17), but it catalyzed the ATPdependent eviction of H2A.Z and promoted H2A incorporation (Fig. 2C). Thus, the activity of the Swc2 $\Delta$ subcomplex shows similarity to that of SWR-C with an H3-K56Q nucleosome. The ATPase activity of the $\mathrm{Swc} 2 \Delta$ subcomplex was stimulated by an H2A.Z nucleosome, but not an H2A nucleosome, the opposite substrates compared to intact SWR-C (Fig. 2D). Thus, the Swc2/3 module appears to play a key role in substrate specificity, promoting activity on an $\mathrm{H} 2 \mathrm{~A}$ nucleosome and preventing the remodeling of an $\mathrm{H} 2 \mathrm{~A} . \mathrm{Z}$ nucleosome. Swc2p binds to the C-terminal domain of H2A.Z that is near H3-K56 within the nucleosome; thus, we propose that H3-K56Ac might disrupt Swc2p function, allowing SWR-C to act on a H2A.Z nucleosome. Consistent with this view, the activity of the Swc2 $\Delta$ subcomplex was not influenced by H3-K56Q (fig. S11).

Our model predicts that constitutive acetylation of H3-K56 in vivo should promote H2A.Z exchange, leading to decreased steady-state levels. To test this model, we carried out genome-wide mapping of H2A.Z in yeast with globally increased levels of H3K56Ac. For these analyses, yeast strains were used that either express H3K56Q, or lack the Hst3p and Hst4p deacetylases that target H3-K56Ac $(18,19)$. In our wild-type control, we recapitulated the previously reported localization of H2A.Z $(1,3)$, with maximal enrichment at genic +1 nucleosomes and more modest enrichment at the -1 nucleosome. Notably, in both strains carrying globally increased H3-K56ac, H2A.Z levels were on average diminished at promoters (Fig. 3A). HTZ1 (H2A.Z) mRNA levels were unchanged in H3-K56Q and hst3L/ hst $4 \Delta$ strains (fig. S12). Because Fig. 3A shows an averaged view over all genes, we also sought to understand whether this loss of $\mathrm{H} 2 \mathrm{~A} . \mathrm{Z}$ was universal or specific to a small subset of genes. As shown in Fig. 3, B and C, H2A.Z was generally lost from +1 nucleosomesnucleosomes normally exhibiting modest or minimal enrichment of H2A.Z (H2A.Z levels in wild type from 0 to 3 ) were little affected by global hyperacetylation, whereas nucleosomes carrying higher amounts of H2A.Z almost universally lost H2A.Z in strains harboring constitutive H3-K56Ac (see also fig. S13). Consistent with the idea that H3K56Ac leads to SWR-C-dependent H2A.Z replacement, H2A.Z was lost in hyperacetylation mutants primarily at genes associated with SWR-C, whereas genes lacking SWR-C in prior mapping studies (20) were largely unaffected in these mutants (Fig. 3D and fig. S13C).

H2A.Z incorporation was also monitored in wild-type and H3-K56Q strains during the reestablishment of transcriptional repression at the $\mathrm{PHO} 5$ gene. In both the wild-type and H3-K56Q strains, H3 levels were restored to similar extents when PHO5 was repressed; however, H2A.Z was at least 50\% less in the H3-K56Q strain (fig. S14). Finally, we tested whether the gene expression profiles of an H3-K56Q strain are similar to that of a strain that lacks H2A.Z. We observed a significant overlap in gene expression defects and a positive correlation between changes in mRNA levels between htz1 $1 \Delta$ and H3K56Q strains (Fig. 4). These data are consistent with H3K56Ac modulating SWR-C dimer-exchange activity in vivo.

A functional connection between histone modifications and ATP-dependent remodeling enzymes has long been recognized (21). Here we find that H3-K56Ac (or H3-K56Q) 
functions as a switch that changes the remodeling specificity of the SWR-C dimer-exchange reaction, leading to removal of H2A.Z from the nucleosomal product. Our data indicate that Swc2p functions to prevent activation of the Swr1 ATPase by an H2A.Z nucleosome, and that it may function at the end of the reaction cycle to "lock" H2A.Z and prevent its eviction. Swc2p is conserved from yeast to human (17), and YL-1, the metazoan counterpart of Swc2p, is found in the Drosophila and human counterparts of SWR-C, the dTip60 and SRCAP complexes, respectively $(22,23)$. Thus, it is likely that both the proposed "lock" function of Swc2 and the role of H3-K56Ac are conserved in higher eukaryotes. Our work suggests a model whereby an H3-K56Ac nucleosome may be subject to multiple rounds of SWR-C-catalyzed dimer exchange and such promiscuous dimer exchange may promote subsequent histone $\mathrm{H} 3 / \mathrm{H} 4$ turnover (fig. S15). This model provides a mechanistic explanation for how H3-K56Ac and H2A.Z might coordinately control nucleosome turnover at regulatory regions (3) by regulating the activity and substrate specificity of chromatin remodeling enzymes.

\section{Supplementary Material}

Refer to Web version on PubMed Central for supplementary material.

\section{Acknowledgments}

We thank P. D. Kaufman (University of Massachusetts Medical School) for the gift of purified Rtt109/Vps75. We apologize to colleagues whose work we could not cite due to space constraints. This work was supported by grants from the NIH to C.L.P. (RO1 GM49650) and to O.J.R. (R01 GM079205). Chip-Seq data sets have been assigned the Gene Expression Omnibus accession no. GSE43935.

\section{References and Notes}

1. Raisner RM, et al. Cell. 2005; 123:233. [PubMed: 16239142]

2. Barski A, et al. Cell. 2007; 129:823. [PubMed: 17512414]

3. Albert I, et al. Nature. 2007; 446:572. [PubMed: 17392789]

4. Dion MF, et al. Science. 2007; 315:1405. [PubMed: 17347438]

5. Deal RB, Henikoff JG, Henikoff S. Science. 2010; 328:1161. [PubMed: 20508129]

6. Rufiange A, Jacques PE, Bhat W, Robert F, Nourani A. Mol Cell. 2007; 27:393. [PubMed: 17679090]

7. Kaplan T, et al. PLoS Genet. 2008; 4:e1000270. [PubMed: 19023413]

8. Xie W, et al. Mol Cell. 2009; 33:417. [PubMed: 19250903]

9. Lo KA, et al. PLoS ONE. 2011; 6:e19778. [PubMed: 21655096]

10. Watanabe S, et al. Biochim Biophys Acta. 2010; 1799:480. [PubMed: 20100606]

11. Neumann H, et al. Mol Cell. 2009; 36:153. [PubMed: 19818718]

12. Zhang H, Roberts DN, Cairns BR. Cell. 2005; 123:219. [PubMed: 16239141]

13. Krogan NJ, et al. Mol Cell. 2003; 12:1565. [PubMed: 14690608]

14. Mizuguchi G, et al. Science. 2004; 303:343. [PubMed: 14645854]

15. Luk E, et al. Cell. 2010; 143:725. [PubMed: 21111233]

16. Papamichos-Chronakis M, Watanabe S, Rando OJ, Peterson CL. Cell. 2011; 144:200. [PubMed: 21241891]

17. Wu WH, et al. Nat Struct Mol Biol. 2005; 12:1064. [PubMed: 16299513]

18. Celic I, et al. Curr Biol. 2006; 16:1280. [PubMed: 16815704]

19. Maas NL, Miller KM, DeFazio LG, Toczyski DP. Mol Cell. 2006; 23:109. [PubMed: 16818235]

20. Koerber RT, Rhee HS, Jiang C, Pugh BF. Mol Cell. 2009; 35:889. [PubMed: 19782036]

21. Pollard KJ, Peterson CL. Bioessays. 1998; 20:771. [PubMed: 9819566]

22. Kusch T, et al. Science. 2004; 306:2084. [PubMed: 15528408] 
23. Cai Y, et al. J Biol Chem. 2005; 280:13665. [PubMed: 15647280] 


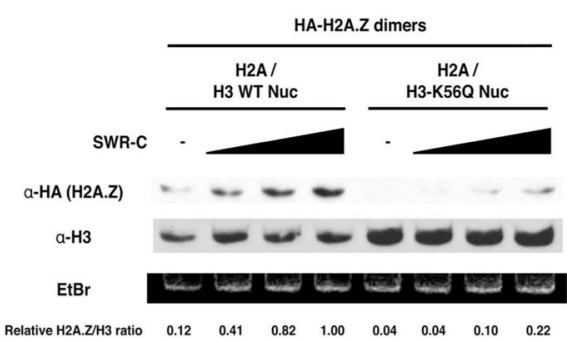

B

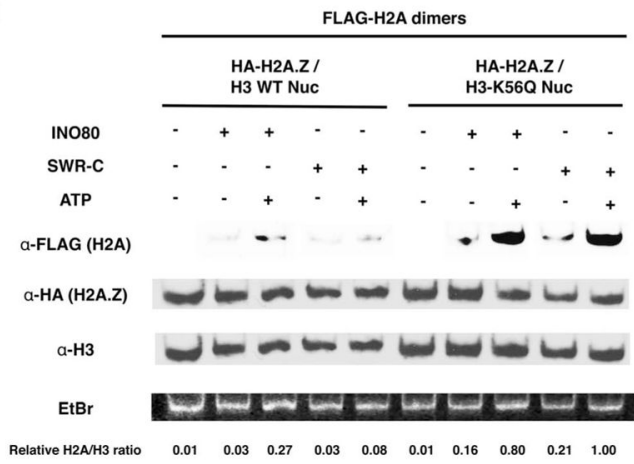

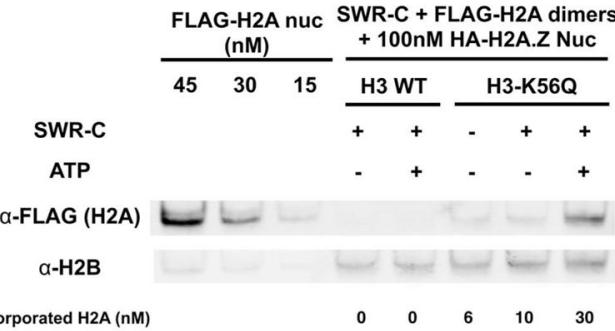

D

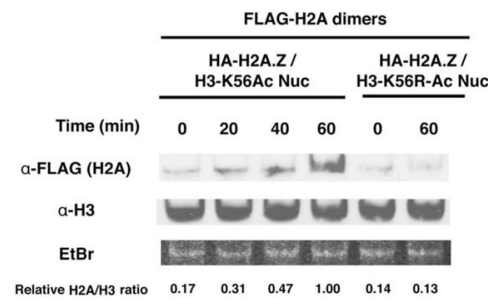

Fig. 1. H3-K56Q alters the substrate specificity of SWR-C dimer exchange (A) Incorporation of H2A.Z by SWR-C on H3-K56Q nucleosomes. Dimer-exchange assay performed with SWR-C (1 to $5 \mathrm{nM})$, HA-H2A.Z/H2B dimers $(50 \mathrm{nM})$, and H2A/H3WT or H2A/H3-K56Q nucleosomes (100 nM) with $1 \mathrm{mM} \mathrm{ATP.} \mathrm{H2A.Z/H3} \mathrm{ratios} \mathrm{were} \mathrm{normalized}$ to lane 4. (B) SWR-C incorporates H2A into H2A.Z/H3-K56Q nucleosomes and H3-K56Q stimulates dimer-exchange activity of INO80. Dimer-exchange assay performed with $5 \mathrm{nM}$ SWR-C or $1 \mathrm{nM}$ INO80, FLAG-H2A/H2B dimers (50 nM), and HA-H2A.Z/H3WT or HAH2A.Z/H3-K56Q nucleosomes (100 nM) with (+) or without (-) $1 \mathrm{mM} \mathrm{ATP.} \mathrm{Each} \mathrm{H2A/H3}$ ratio was normalized to lane 10 . (C) SWR-C-mediated deposition of $\mathrm{H} 2 \mathrm{~A}$ quantified by quantitative western blotting (LI-COR). Dimer-exchange assays performed with $10 \mathrm{nM}$ SWR-C, FLAG-H2A/H2B dimer and NAP1 (200 nM), and HA-H2A.Z/H3WT or HAH2A.Z/H3-K56Q nucleosomes (100 nM) with or without $1 \mathrm{mM} \mathrm{ATP.} \mathrm{(D)} \mathrm{H3K56Ac}$ stimulates $\mathrm{H} 2 \mathrm{~A}$ deposition by SWR-C. Dimer-exchange assay performed with SWR-C (5 nM), ATP (1 mM), FLAG-H2A/H2B dimers (50 nM), and HA-H2A.Z/H3-K56Ac or HAH2A.Z/H3-K56R-Ac nucleosomes (100 nM) generated by in vitro acetylation with Rtt109/ Vps75 acetyltransferase. EtBr, ethidium bromide. 


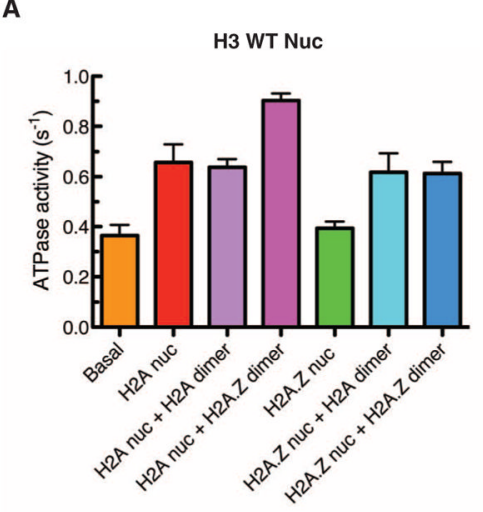

C

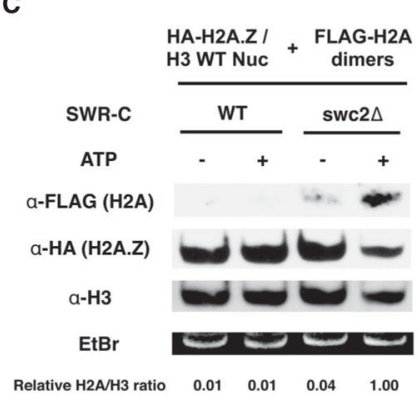

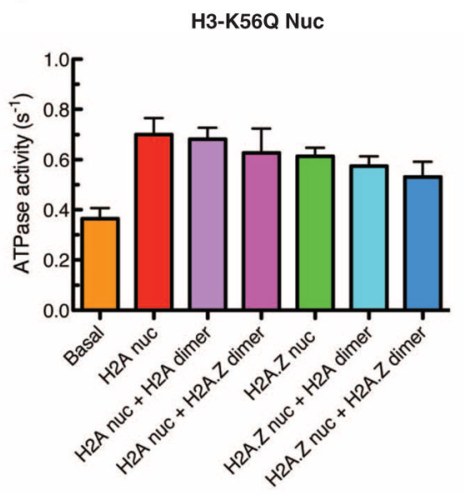

D

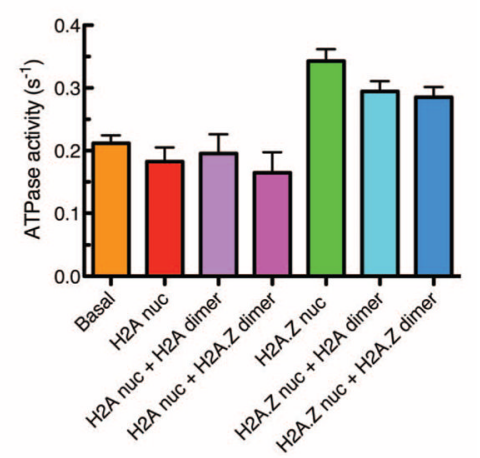

Fig. 2. Roles for H3-K56Q and Swc2p in modulating the ATPase cofactor requirements of SWRC

(A) Substrate specificity of SWR-C ATPase activity on H3WT nucleosomes. SWR-C (1 $\mathrm{nM})$ and $0.1 \mathrm{mM}$ ATP was incubated with $(+)$ or without $(-)$ nucleosomes $(15 \mathrm{nM})$ or free dimers (15 nM). ATPase assays measured fluorescent change of 7-diethylamino-3-(\{[(2maleimidyl)-ethyl]amino \} carbonyl)coumarin-labeled phosphate-binding protein (MDCCPBP) upon phosphate binding. Data represent the results from three independent experiments and error bars reflect standard deviations. (B) Substrate specificity of ATPase activity of SWR-C on H3-K56Q nucleosomes. As in (A), but nucleosomes harbored H3K56Q. (C) The Swc2 $\Delta$ subcomplex exchanges H2A.Z with H2A. The dimer-exchange assay performed with $5 \mathrm{nM}$ SWR-C WT or Swc2 $\Delta$ subcomplex, FLAG-H2A/H2B dimers $(50 \mathrm{nM})$, and HA-H2A.Z/H3WT nucleosomes $(100 \mathrm{nM})$ with or without $1 \mathrm{mM}$ ATP. Each $\mathrm{H} 2 \mathrm{~A} / \mathrm{H} 3$ ratio was normalized to lane 4 . EtBr, ethidium bromide. (D) Substrate specificity of the $\operatorname{Swc} 2 \Delta$ subcomplex. 


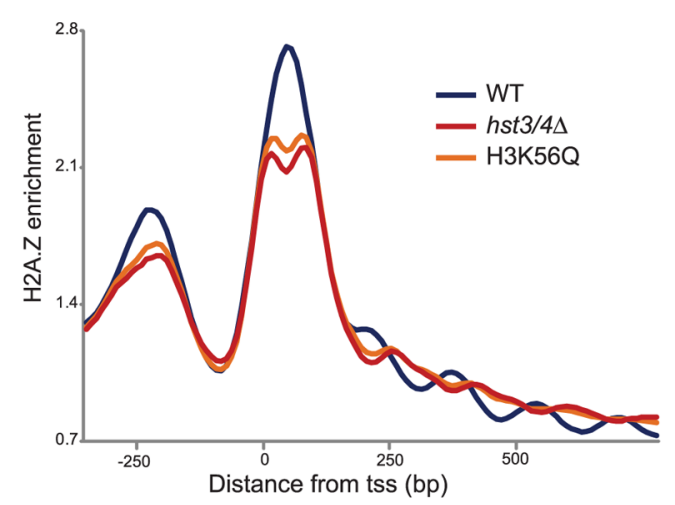

C

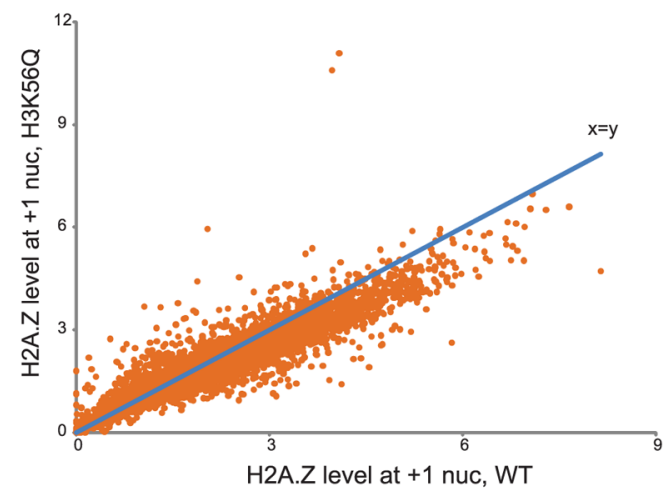

B

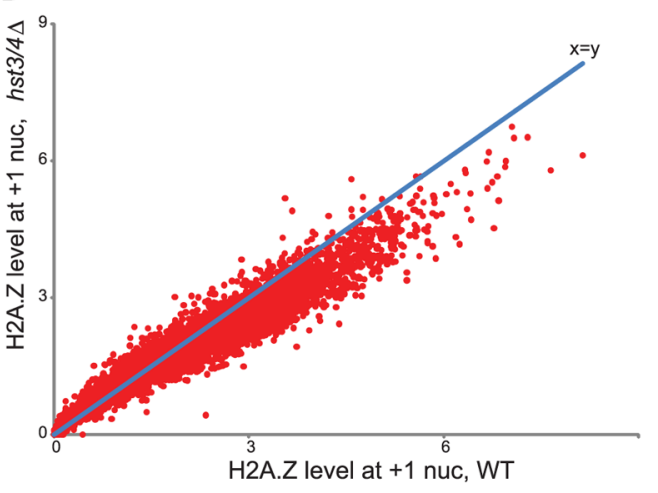

D

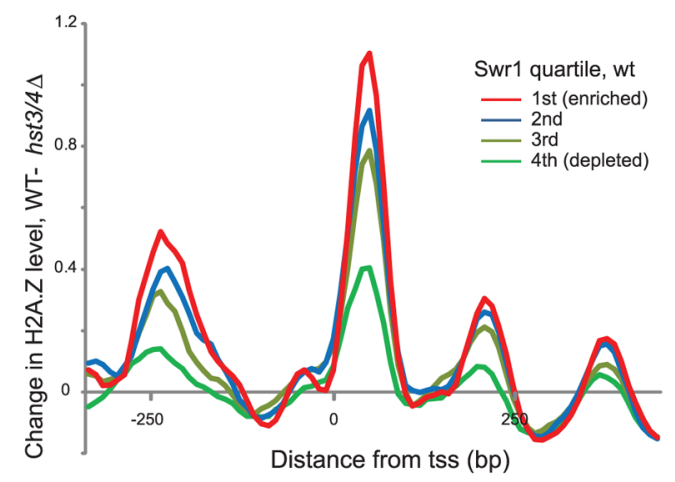

Fig. 3. Constitutive H3-K56Ac decreases steady-state levels of promoter-proximal H2A.Z in vivo (A) H2A.Z enrichments at promoter regions in wild-type (WT) and H3-K56Q strains. Chromatin immunoprecipitation sequencing (ChIP-Seq) using antibody against H2A.Z in WT, hst3 $\Delta$ hst4 , and H3K56Q strains. After alignment to the genome, normalized read counts were averaged for all genes aligned on the basis of their transcriptional start sites (TSS). (B and C) Scatterplot of H2A.Z levels at genic +1 nucleosomes for WT ( $x$ axis) versus either hst3 $\Delta$ hst4 $\Delta$ (B) or H3K56Q (C) mutants. Blue lines show $x=y$ line. The vast majority of genes exhibit a decrease in H2A.Z levels in either of the H3K56 hyperacetylated mutants. (D) H2A.Z loss preferentially occurs at genes associated with the SWR-C complex. Swr1 levels [from (20)] were calculated for all genes, and genes are grouped into four quartiles according to Swr1 abundance. Change in H2A.Z levels at the +1 nucleosome in WT and hst $3 \Delta$ hst $4 \Delta$ strains is shown at promoters, showing that genes with the lowest Swr1 levels exhibited lower changes in H2A.Z levels than remaining genes with moderate to high Swr1 levels. 


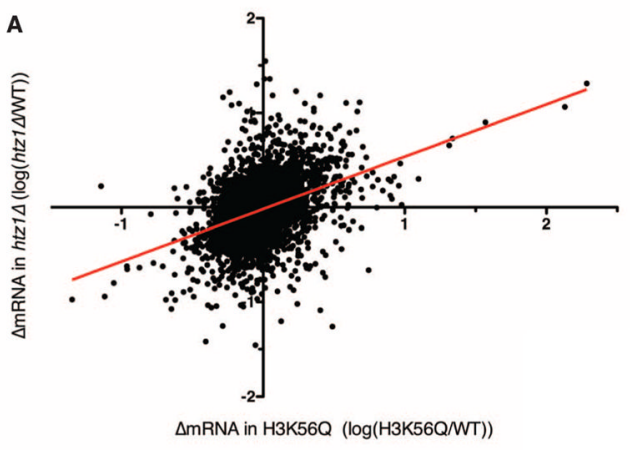

B

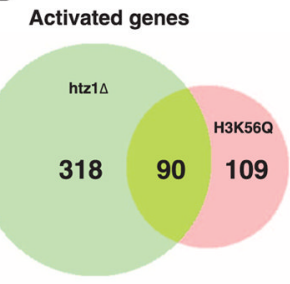

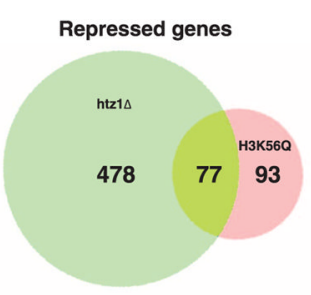

Fig. 4. Positive correlation of gene expression profiles between H3-K56Q and $h t z 1 \Delta$ strains (A) Scatterplot analysis of change in RNA level measured as the $\log _{2}$ of mutant/WT expression ratio. Gene microarray analysis was conducted in the $H 3-K 56 Q$ and $h t z 1 \Delta$ strains cultured in YPD media at $30^{\circ} \mathrm{C}$. $R=0.393$. (B) Venn diagrams showing the number of genes for which RNA levels changed by 1.25 -fold in mutants relative to the WT strain. $P$ values were $8 \times 10^{-54}$ (45\% overlap in activated genes) and $9 \times 10^{-36}(45 \%$ overlap in repressed genes). 